Pacific

Journal of

Mathematics

THE DISTRIBUTION OF JAGER PAIRS FOR CONTINUED FRACTION LIKE MAPPINGS OF THE INTERVAL

Andrew HaAs and David Molnar

Volume 217 No. 1

November 2004 


\title{
THE DISTRIBUTION OF JAGER PAIRS FOR CONTINUED FRACTION LIKE MAPPINGS OF THE INTERVAL
}

\author{
ANDREw HaAs AND DAVID MOLnaR
}

\begin{abstract}
Certain ergodic, piecewise Möbius self-mappings of the unit interval, similar to the classical Gauss or Rényi maps, give rise to natural sequences of convergents $p_{n} / q_{n}$ for every associated "irrational" number $x$. Here we study the metric theory of the approximation sequences $\theta_{n}=\left|q_{n}\right|\left|q_{n} x-p_{n}\right|$. Following Jager we describe the distribution of pairs $\left(\theta_{n}, \theta_{n+1}\right)$ in a plane domain by deriving their distribution function. As a consequence we get a generalization of the theorem of Bosma, Jager and Wiedijk, referred to as the Lenstra Conjecture, which describes the distribution of the $\theta_{n}$.
\end{abstract}

\section{Introduction}

It is a well-known fact that every irrational number $x \in(0,1)$ admits a unique representation as a continued fraction of the form

$$
\left[n_{1}, n_{2}, n_{3}, \ldots\right]=\frac{1}{n_{1}+\frac{1}{n_{2}+\frac{1}{n_{3}+\cdots}}},
$$

where the $n_{i}$ are positive integers. The expansion determines an infinite sequence of rational fractions $p_{i} / q_{i}=\left[n_{1}, n_{2}, \ldots, n_{i}\right]$, in lowest terms, known as the convergents of $x$.

One important approach for studying the classical theory is by way of the Gauss map

$$
G(x)=\frac{1}{x}-\left[\frac{1}{x}\right]=\left\langle\frac{1}{x}\right\rangle,
$$

where $[a]$ denotes the integral part of $a$ and $\langle a\rangle$ the fractional part. For technical reasons define the fractional part of infinity to be zero. If we let $G^{i}$ denote the $i$-th iterate of $G$, the continued fraction expansion of $x \in(0,1)$ has terms $n_{i}=\left[1 / G^{i-1}(x)\right]$.

The Gauss map is an example of an ergodic, piecewise Möbius mapping of the unit interval. With such a mapping $T$, one comes to expect an associated continued fraction theory. The digits in the expansion of $x$ correspond to 
the sequence of intervals of monotonicity of the iterates $T^{n}$ nesting about $x$, while certain endpoints of the intervals of monotonicity can be viewed naturally as "fractions" $p_{n} / q_{n}$, corresponding to the convergents of $x$.

Given a Möbius transformation $A$ that takes one of the endpoints $\{0,1\}$ to 0 and the other to $\infty$, define the transformation

$$
T(x)=\langle A(x)\rangle .
$$

On a one-parameter family the maps have finite invariant measures, equivalent to Lebesgue measure, with respect to which they are ergodic. In their graphical appearance as well as in the dynamic interval structures they define, these maps are virtually identical to either the Gauss map or the closely related Rényi or backwards continued fraction map [7].

As above, for $x \in(0,1)$, the sequence of integers $a_{i}=\left[A\left(T^{i-1}(x)\right)\right]$, when defined, determines a continued fraction expansion for $x$ with respect to the particular Möbius transformation $A$. For example, on a one-parameter subfamily particularly resembling the Gauss map the representations are of the form

$$
x=\frac{t}{\frac{a_{1}}{t}+t+\frac{1}{\frac{a_{2}}{t}+t+\frac{1}{\frac{a_{3}}{t}+t+\cdots}}}
$$

where $t>0$ and the $a_{i}$ are now nonnegative integers. In this setting the classical theory obviously corresponds to the choice of parameter $t=1$.

The expansion of $x$ also determines a sequence of convergents $p_{n} / q_{n}$, converging to $x$. One important measure of the degree to which a convergent approximates $x$ is given in classical form by the function $\theta_{n}(x)$ defined by the identity

$$
\left|x-\frac{p_{n}}{q_{n}}\right|=\frac{\theta_{n}(x)}{q_{n}^{2}} .
$$

In [9] we developed the basic theory as well as many metrical results for the continued fractions described above. In this paper we shall focus more sharply on the metrical theory of the sequences of approximation values $\theta_{n}$. Following Jager [10], each Möbius transformation $A$, as above, is shown to determine a plane domain $\Omega$ and a density $\lambda$ such that for almost all $x \in(0,1)$ the sequence of Jager pairs $\left(\theta_{n}, \theta_{n+1}\right)$ is distributed in $\Omega$ according to the density $\lambda$. These objects are described quite explicitly as a function of the transformation $A$. As a consequence we get a generalization of the theorem of Bosma, Jager and Wiedijk from [5], referred to as the Lenstra Conjecture, which describes the distribution of the $\theta_{n}$. These results are applied to the computation of the generic limiting averages of the values $\theta_{n}$ and $\left|\theta_{n}-\theta_{n+1}\right|$. 
It is also interesting to note that given a Möbius transformation $A$, as above, the shift map $\left(\theta_{n}, \theta_{n+1}\right) \rightarrow\left(\theta_{n+1}, \theta_{n+2}\right)$ extends to an automorphism of $\Omega$ for which the density $\lambda$ is invariant. We prove that this dynamical system is Bernoulli.

\section{Preliminaries}

\section{1. u-continued fraction expansions and the generalized Gauss}

map. We begin with a review of the basic continued fraction theory developed in [8]. For $k \neq 0$ consider the Möbius transformations

$$
A_{(k, 0)}(z)=\frac{k(1-z)}{z} \text { and } A_{(k, 1)}(z)=\frac{k z}{1-z} .
$$

Möbius transformations like these, with real coefficients, preserve the extended real line $\mathbb{R} \cup\{\infty\}$. The Möbius transformations $A$ described in the introduction are precisely the $A_{u}(z)$ with $u \in \Psi=\Psi_{0} \cup \Psi_{1}$, where

$$
\Psi_{0}=((-\infty,-1) \cup(0, \infty)) \times\{0\} \quad \text { and } \quad \Psi_{1}=((-\infty, 0) \cup(1, \infty)) \times\{1\} .
$$

It will often be more convenient to use following real matrices of determinant \pm 1 to represent the transformations:

$$
A_{(k, 0)}=\left(\begin{array}{cc}
\frac{k}{\sqrt{|k|}} & \frac{-k}{\sqrt{|k|}} \\
\frac{-1}{\sqrt{|k|}} & 0
\end{array}\right) \quad \text { and } \quad A_{(k, 1)}=\left(\begin{array}{cc}
\frac{k}{\sqrt{|k|}} & 0 \\
\frac{-1}{\sqrt{|k|}} & \frac{1}{\sqrt{|k|}}
\end{array}\right) .
$$

The product of a pair of matrices represents the composition of the corresponding transformations. We shall also need the matrix $B=\left(\begin{array}{ll}1 & 1 \\ 0 & 1\end{array}\right)$ in our work. See [1] for the basics on Möbius transformations.

For $u \in \Psi$, define the set of integers $V_{u}$, called the $u$-digits, to be the set of nonnegative integers if $k>0$ and the set of negative integers if $k<0$. Given a finite sequence of $u$-digits $a_{1}, a_{2}, \ldots, a_{n}$, define the finite $u$-continued fraction expansion

$$
\left[a_{1}, a_{2}, \ldots, a_{n}\right]_{u}=A_{u}^{-1} B^{a_{1}} A_{u}^{-1} B^{a_{2}} \ldots A_{u}^{-1} B^{a_{n}} A_{u}^{-1}(\infty) \in[0,1] .
$$

In particular, if we write

$$
A_{u}^{-1} B^{a_{1}} A_{u}^{-1} B^{a_{2}} \ldots A_{u}^{-1} B^{a_{n}} A_{u}^{-1}=\left(\begin{array}{cc}
p_{n} & r_{n} \\
q_{n} & s_{n}
\end{array}\right),
$$

then $\left[a_{1}, a_{2}, \ldots, a_{n}\right]_{u}=p_{n} / q_{n}$.

Write $I=[0,1]$ for the closed unit interval. For $u \in \Psi$, the interval transformation $T_{u}: I \rightarrow I$ is the piecewise Möbius transformation $T$ defined in Equation (1) with $A=A_{u}$. This transformation generalizes the classical Gauss map, with which it agrees when $u=(1,0)$. When $u \in \Psi_{0}$ we shall refer to $T_{u}$ or $u$ itself as Gauss-like, and when $u \in \Psi_{1}$ we shall refer to it as Rényi-like. 
2.2. The basic facts. The basic facts about the representation of real numbers by $u$-continued fraction expansions and the transformations $T_{u}$ are collected from $[\mathbf{8}]$ and $[\mathbf{9}]$.

1. Given an infinite sequence of $u$-digits $\left\{a_{n}\right\}_{n=1}^{\infty}$, there is a unique $x \in(0,1)$ with $x=\lim _{n \rightarrow \infty}\left[a_{1}, a_{2}, \ldots, a_{n}\right]_{u}=\lim _{n \rightarrow \infty} p_{n} / q_{n}$.

A number $x \in[0,1]$ is $u$-rational if $T_{u}^{n}(x)=0$ for some positive integer $n$. Such numbers form a countable set denoted by $\mathbb{Q}_{u}$. The complement of $\mathbb{Q}_{u}$ is the set of $u$-irrational numbers.

2. Every $u$-irrational number has a unique infinite $u$-expansion. If the irrational $x$ has the expansion $\left[a_{1}, a_{2}, \ldots\right]_{u}$ then $T(x)=\left[a_{2}, a_{3}, \ldots\right]_{u}$.

For each $u=(k, m) \in \Psi$ define the intervals

$$
J_{u}= \begin{cases}(-\infty,-k] & \text { if } m=0 \text { and } k>0, \\ {[-k, \infty)} & \text { if } m=0 \text { and } k<-1, \\ (-\infty, 1-k] & \text { if } m=1 \text { and } k>1, \\ {[1-k, \infty)} & \text { if } m=1 \text { and } k<0\end{cases}
$$

Set $\rho_{u}(x, y)=c_{u}(x-y)^{-2}$, where $c_{u}=\operatorname{sgn}(k)\left(\log \left|\frac{k+1-m}{k-m}\right|\right)^{-1}$. Given a Borel subset $D$ of $I \times J_{u}$,

$$
\rho_{u}(D)=\iint_{D} \rho_{u}(x, y) d x d y
$$

defines a Borel probability measure on $I \times J_{u}$.

3. The map $\widetilde{T}_{u}: I \times J_{u} \rightarrow I \times J_{u}$ defined by

$$
\widetilde{T}_{u}(x, y)=\left(T_{u}(x), A_{u}(y)-\left\langle A_{u}(x)\right\rangle\right)
$$

has invariant measure $\rho_{u}$. On the complement of a countable subset, $\widetilde{T}_{u}$ is a Bernoulli automorphism. Consequently, $\widetilde{T}_{u}$ is ergodic. The map $\widetilde{T}_{u}$ is referred to as the natural automorphic extension of $T_{u}$.

Fix $u \in \Psi$. Given $x \in(0,1)$ let

$$
\left(x_{n}, y_{n}\right)=\widetilde{T}_{u}^{n+1}(x, \infty) .
$$

If $x$ is $u$-irrational, $\left(x_{n}, y_{n}\right)$ is defined and lies in $I \times J_{u}$ for all integers $n \geq 0$.

4. In terms of the natural extension we have

$$
\theta_{n}(x)=\left|x_{n}-y_{n}\right|^{-1} \text {. }
$$

A variant of the ergodicity of $\widetilde{T}$, which is very important for the proofs in this paper, is given in the following theorem from [9]:

5. For $u \in \Psi$ and for almost all $x \in[0,1]$, the points $\widetilde{T}_{u}^{n}(x, y)$ for integers $n \geq 0$ are distributed in the interior of the region $I \times J_{u}$ according to the 
density function $\rho_{u}(x, y)$ for all $y \in J_{u} \cup\{\infty\}$. In other words, for any Borel set $D \subset I \times J_{u}$ having boundary of measure zero and for almost all $x$,

$$
\lim _{n \rightarrow \infty} \frac{1}{n} \#\left\{j \leq n \mid \widetilde{T}_{u}^{n}(x, y) \in D\right\}=\rho_{u}(D)
$$

for all $y \in J_{u} \cup\{\infty\}$. Furthermore, for any uniformly continuous function $f \in L^{1}\left(\rho_{u}\right)$ and for almost all $x \in I$,

$$
\lim _{n \rightarrow \infty} \frac{1}{n} \sum_{i=1}^{n} f\left(\widetilde{T}_{u}^{n}(x, y)\right)=\int f d \rho_{u}
$$

for all $y \in J_{u} \cup\{\infty\}$.

\section{Approximating pairs $\left(\theta_{n}, \theta_{n+1}\right)$}

3.1. $\left(\theta_{\mathrm{n}}, \boldsymbol{\theta}_{\mathrm{n}+1}\right)$ as a function of $(x, y) \in I \times J_{u}$. As a consequence of the relationship expressed in Equation (4), it is possible to study measure theoretic properties of the approximation sequence $\left\{\theta_{n}\right\}$ defined in (2) for a given $u$-irrational, by way of the natural extension. As in Jager [10] our approach shall be to begin with the even richer sequence of approximating pairs $\left(\theta_{n}, \theta_{n+1}\right)$.

Define the functions

$$
F_{u}(x, y)=\operatorname{sgn}(k)\left(\frac{1}{x-y}, \frac{x y}{k(y-x)}\right)
$$

when $u$ is Gauss-like and

$$
F_{u}(x, y)=\operatorname{sgn}(k)\left(\frac{1}{x-y}, \frac{(1-x)(1-y)}{k(x-y)}\right)
$$

when $u$ is Rényi-like.

Let $\hat{\mathbb{R}}^{2}$ be the set $\mathbb{R}^{2}$ with the points in the diagonal set $\{(x, y) \mid x=y\}$ deleted. For each $u \in \Psi$, the map $F_{u}: \hat{\mathbb{R}}^{2} \rightarrow \mathbb{R}^{2}$ is $C^{\infty}$. As the next proposition makes clear, a basic understanding of these maps provides the connection between the approximating pairs and our earlier work with the natural extension.

Proposition 1. For $u \in \Psi$ and $x$ u-irrational, $\left(\theta_{n}, \theta_{n+1}\right)=F_{u}\left(x_{n}, y_{n}\right)$.

Proof. A straightforward computation using Fact 4, formula (3) and the definition of $A_{u}$ gives

$$
\begin{aligned}
\left(\theta_{n}, \theta_{n+1}\right) & =\left(\frac{1}{\left|x_{n}-y_{n}\right|}, \frac{1}{\left|x_{n+1}-y_{n+1}\right|}\right) \\
& =\left(\frac{1}{\left|x_{n}-y_{n}\right|}, \frac{1}{\left|\left(A_{u}\left(x_{n}\right)-\left[A_{u}\left(x_{n}\right)\right]\right)-\left(A_{u}\left(y_{n}\right)-\left[A_{u}\left(x_{n}\right)\right]\right)\right|}\right) \\
& =\left(\frac{1}{\left|x_{n}-y_{n}\right|}, \frac{1}{\left|A_{u}\left(x_{n}\right)-A_{u}\left(y_{n}\right)\right|}\right)=F_{u}\left(x_{n}, y_{n}\right) .
\end{aligned}
$$


The set $\hat{\mathbb{R}}^{2}$ has two connected components, $U$ (for upper) with $x<y$ and $L$ with $x>y$. $U$ and $L$ may be further divided, in a way depending on $u$, into two pieces. When $u$ is Gauss-like $U_{u}^{+} \subset U$ and $L_{u}^{-} \subset L$ have $-x \geq y$ while $U_{u}^{-} \subset U$ and $L_{u}^{+} \subset L$ have $-x \leq y$. When $u$ is Rényi-like $U_{u}^{+} \subset U$ and $L_{u}^{-} \subset L$ have $-x+2 \geq y$ while $U_{u}^{-} \subset U$ and $L_{u}^{+} \subset L$ have $-x+2 \leq y$.

Theorem 1. The transformation $F_{u}$ is injective on each of the sets $U_{u}^{+}, U_{u}^{-}$, $L_{u}^{+}$and $L_{u}^{-}$. The inverses are

$$
x=\frac{1 \pm \sqrt{1-4 k w z}}{\operatorname{sgn}(k) 2 w} \quad \text { and } \quad y=\frac{-1 \pm \sqrt{1-4 k w z}}{\operatorname{sgn}(k) 2 w}
$$

for Gauss-like maps and

$$
x=\frac{2 w+1 \pm \sqrt{1-4 k w z}}{\operatorname{sgn}(k) 2 w} \quad \text { and } \quad y=\frac{2 w-1 \pm \sqrt{1-4 k w z}}{\operatorname{sgn}(k) 2 w}
$$

for Rényi-like maps, where the \pm sign before the square root matches the superscript sign in the symbol for the set.

Proof. The proof for the sets $L_{u}$ is much the same as that for the sets $U_{u}$, which follows.

First suppose $u$ is Gauss-like. The transformation $\sigma_{u}(x, y)=(-y,-x)$ is an order-two involution interchanging $U^{+}$and $U^{-}$. Also,

$$
F_{u}(-y,-x)=F_{u}(x, y) .
$$

So it suffices to restrict our attention to $U^{+}$. Computing the Jacobian derivative of $F_{u}$ we get

$$
\frac{2(x+y)}{k(x-y)^{3}}
$$

which vanishes only on the boundary $\{(x, y) \mid y=-x\}$ in $U^{+}$.

Next, setting

$$
w=\frac{1}{x-y} \quad \text { and } \quad z=\frac{x y}{k(y-x)}
$$

and solving for $z$ and $w$ gives formulae (5). Since $F_{u}$ is continuous in the interior of the connected set $U^{+}$, the \pm in front of the square root can only change from one sign to the other at points in the $w, z$-plane with $1-4 k w z=0$. This set is the image of the solution to $-4 x y /(x-y)^{2}=1$ or $y=-x$. It follows that $F_{u}$ is invertible on $U^{+}$and $U^{-}$. By checking the maps at a single point, for example $(-1,0)$, it is seen that the inverse is given by (5) with the minus sign on $U^{-}$and with the plus sign on $U^{+}$.

Now suppose instead that $u$ is Rényi-like. The transformation $\sigma_{u}(x, y)=$ $(-y+2,-x+2)$ is an order-two involution interchanging $U_{u}^{+}$and $U_{u}^{-}$and $F_{u}(-y+2,-x+2)=F_{u}(x, y)$. As above the Jacobian derivative of $F_{u}$ vanishes only on $\{(x, y) \mid y=-x+2\}$. 
Solving for the inverse map we get formulae (6). As above $F_{u}$ is invertible on the two subsets of $U_{u}$ and the inverse is given by (6) with the minus sign on $U_{u}^{-}$and with the plus sign on $U_{u}^{+}$.

Remark. The proof above also shows that, given $(x, y) \in \hat{\mathbb{R}}^{2}$, we have $F(x, y)=F\left(x^{\prime}, y^{\prime}\right)$ if and only if either $\sigma(x, y)=\left(x^{\prime}, y^{\prime}\right)$ or $(x, y)=\left(x^{\prime}, y^{\prime}\right)$.

3.2. The space of approximating pairs $\boldsymbol{\Omega}_{\boldsymbol{u}}=\boldsymbol{F}_{\boldsymbol{u}}\left(\boldsymbol{I} \times \boldsymbol{J}_{\boldsymbol{u}}\right)$. In $\mathbb{R}^{2}$ consider the points $O=(0,0), P_{u}=(1 /|k|, 0), R_{u}=(0,1 /|k|)$ and

$$
Q_{u}= \begin{cases}\left(\frac{1}{|k|+1}, \frac{1}{|k|+1}\right) & \text { if }(-1)^{m} k>0, \\ \left(\frac{1}{|k|-1}, \frac{1}{|k|-1}\right) & \text { if }(-1)^{m} k<0 .\end{cases}
$$

Let $\Omega_{u}^{*}$ denote the quadrilateral with vertices $O, P_{u}, Q_{u}, R_{u}$ in the $w, z$-plane, from which the line segment from $R_{u}$ to $O$ has been deleted. For $|k|<1$ define $\Lambda_{u}$ as the closed region bounded by the lines $\overline{P_{u} Q_{u}}$ and $\overline{Q_{u} R_{u}}$ and the hyperbola $z=1 /(4|k| w)$. Let $\Lambda_{u}=\emptyset$ when $|k| \geq 1$. Define $\Omega_{u}=\Omega_{u}^{*} \cup \Lambda_{u}$.
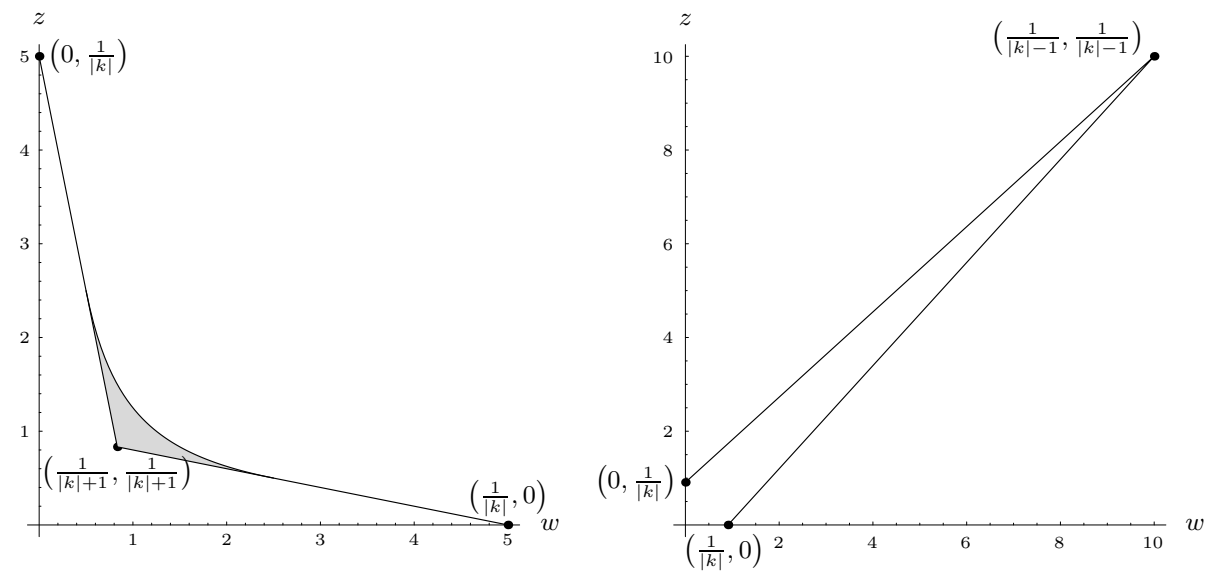

Figure 1. $\Omega_{u}$ for the cases $k=\frac{1}{5}$ and $k=-\frac{11}{10}$. The shaded region is $\Lambda_{u}$.

Let $\Gamma_{u}$ be the square in the $x, y$-plane with vertices $(0,1-k),(1-k, 1+k)$, $(k+1,2)$ and $(0,2)$ when $m=1$ and $-1<k<0$ and the square with vertices $(-k,-k),(1,-k),(1,-1)$ and $(-k,-1)$ when $m=0$ and $0<k<1$. Observe that $\sigma_{u}$ acts as an order-two self-map of $\Gamma_{u}$.

In light of the Theorem 1, the map $F_{u}: I \times J_{u} \rightarrow \mathbb{R}^{2}$ can be completely characterized.

Corollary 1. If $|k|>1$ then $F_{u}$ is a homeomorphism from $I \times J_{u}$ to $\Omega_{u}$. If $|k|<1$ then $F_{u}: \Gamma_{u} \rightarrow \Lambda_{u}$ is two-to-one with $F(x, y)=F\left(\sigma_{u}(x, y)\right)$ and 
$F_{u}:\left(I \times J_{u}\right)-\Gamma_{u} \rightarrow \Omega_{u}-\Lambda_{u}$ is a homeomorphism. In particular, for any $u \in \Psi$ and $u$-irrational $x$ the approximating pairs $\left(\theta_{n}, \theta_{n+1}\right)$ belong to $\Omega_{u}$.

Proof. When $|k|>1, I \times J_{u}$ lies entirely in one of the regions $U^{-}$or $L^{-}$. Therefore $F_{u}$ maps $I \times J_{u}$ homeomorphically onto its image. The three boundary arcs of $I \times J_{u}$ map onto the three boundary arcs of $\Omega$ and no points in $I \times J_{u}$ can take values on the omitted boundary arc from $R_{u}$ to $O$. It follows that $F_{u}$ maps $I \times J_{u}$ onto a subset of $\Omega_{u}$. Conversely, $F_{u}^{-1}$ is welldefined in the region lying between the curves $w=0$ and $z=1 /(4|k| w)$ and maps the boundary arcs of $\Omega_{u}$ back where they came from. As above $F_{u}^{-1} \Omega_{u}$ must be a subset of $I \times J_{u}$ and we conclude that $F_{u}$ is a homeomorphism onto $\Omega_{u}$.

Suppose that $|k|<1$. When $m=0$ the line $y=-x$ and when $m=1$ the line $y=-x+2$ will divide $I \times J_{u}$ into a triangle and an unbounded region. By Theorem $1, F_{u}$ is injective on the closure of both pieces. Arguing as above, the closure of the unbounded region maps homeomorphically onto $\Omega_{u}$ while the closure of the triangle maps homeomorphically onto $\Lambda_{u}$. The union of the closed triangle with its image under $\sigma_{u}$ is precisely the square $\Gamma_{u}$.

3.3. The distribution of approximating pairs. It is now an easy matter to prove one of the main theorems of the paper. Define the function

$$
\lambda_{u}(w, z)= \begin{cases}\frac{|k| c_{u}}{\sqrt{1+(-1)^{m+1} 4 k z w}} & \text { if }(w, z) \in \Omega_{u}^{*}, \\ \frac{2|k| c_{u}}{\sqrt{1+(-1)^{m+1} 4 k z w}} & \text { if }(w, z) \in \Lambda_{u} .\end{cases}
$$

We shall abuse notation by also using $\lambda_{u}$ to denote the measure with

$$
\lambda_{u}(D)=\int_{D} \lambda_{u}(w, z) d \mu,
$$

where $D$ is a Borel set and $\mu$ is two-dimensional Lebesgue measure.

Lemma 1. Let $F_{u}^{*} \rho_{u}$ be the Borel probability measure induced by $F_{u}$ on $\Omega_{u}$ by setting $F_{u}^{*} \rho_{u}(D)=\rho_{u}\left(F_{u}^{-1}(D)\right)$. Then $F_{u}^{*} \rho_{u}=\lambda_{u}$.

Proof. The measure is determined by its value on open sets. On an open set $D$ in the interior of $\Omega_{u}^{*}$ we compute

$$
\begin{aligned}
F_{u}^{*} \rho_{u}(D) & =c_{u} \iint_{F_{u}^{-1} D} \frac{d x d y}{(x-y)^{2}} \\
& =c_{u} \iint_{D} f\left(F_{u}^{-1}(w, z)\right)\left|\operatorname{Jac} F_{u}^{-1}(w, z)\right| d w d z,
\end{aligned}
$$


where $f(x, y)=1 /(x-y)^{2}$. From the definition of $F_{u}$ we have $1 /(x-y)^{2}=$ $w^{2}$. The Jacobian is computed from Theorem 1 as

$$
\left|\operatorname{Jac}_{u}^{-1}(w, z)\right|=\frac{|k|}{w^{2} \sqrt{1+(-1)^{m+1} 4 k z w}} .
$$

Thus the second integral in (7) is simply $\lambda_{u}(D)$. Similarly, for open $D$ in the interior of $\Lambda_{u}$ the second integral of (7) is doubled, since the map is two-to-one. The lemma follows.

It is interesting to note at this point that one can induce a Bernoulli automorphism of the space $\Omega_{u}$, which is a type of shift map on the approximating pairs. Suppose that $|k|>1$. Then $F_{u}: I \times J_{u} \rightarrow \Omega_{u}$ is a homeomorphism, so we can define the map $\widetilde{S}_{u}: \Omega_{u} \rightarrow \Omega_{u}$ by $\widetilde{S}_{u}=F_{u} \circ \widetilde{T}_{u} \circ F_{u}^{-1}$. Because of the way we have defined the measure $\lambda_{u}, F_{u}$ is an isomorphism of probability spaces and $\widetilde{S}_{u}$ is a measure preserving transformation of $\Omega_{u}$. Moreover, the dynamical system $\left(I \times J_{u}, \widetilde{T}_{u}, \rho_{u}\right)$ is isomorphic by this conjugacy to the system $\left(\Omega_{u}, \widetilde{S}_{u}, \lambda_{u}\right)$. This proves the following:

Proposition 2. The dynamical system $\left(\Omega_{u}, \widetilde{S}_{u}, \lambda_{u}\right)$ is Bernoulli.

Note that if $(w, z)=\left(\theta_{n}, \theta_{n+1}\right)$ for some $u$-irrational $x$, then

$$
\widetilde{S}_{u}\left(\theta_{n}, \theta_{n+1}\right)=\left(\theta_{n+1}, \theta_{n+2}\right)
$$

is the shift map.

Being Bernoulli, the system $\left(\Omega_{u}, \widetilde{S}_{u}, \lambda_{u}\right)$ is ergodic. In the spirit of Jager [10], we use Fact 5 from Section 2.2 to describe the distribution of pairs as a function of $x$. Although the statement focuses on the distribution of the sequence of pairs, the theorem is easily seen to hold in the context of the dynamical system $\left(\Omega_{u}, \widetilde{S}_{u}, \lambda_{u}\right)$.

Theorem 2. For $u \in \Psi$ and for almost all $x \in[0,1]$ the sequence $\left(\theta_{n}, \theta_{n+1}\right)$, $n=1,2, \ldots$ is distributed in the interior of the region $\Omega_{u}$ according to the density function $\lambda_{u}(w, z)$. In other words, for almost all $x \in(0,1)$ and for any Borel subset $D$ of $\Omega_{u}$ with boundary of measure zero

$$
\lim _{n \rightarrow \infty} \frac{1}{n} \#\left\{j \leq n \mid\left(\theta_{j}, \theta_{j+1}\right) \in D\right\}=\lambda_{u}(D) .
$$

Consequently, for a bounded, uniformly continuous function $f$ and almost all $x \in(0,1)$

$$
\lim _{n \rightarrow \infty} \frac{1}{n} \sum_{j=1}^{n} f\left(\theta_{j}, \theta_{j+1}\right)=\int_{\Omega_{u}} f d \lambda_{u} .
$$

Proof. As a consequence of Fact 5, for almost all $u$-irrational $x$ and for any $y \in J_{u} \cup\{\infty\}$ the sequence $\widetilde{T}_{u}^{n}(x, y)$ is distributed in $I \times J_{u}$ according to the density $\rho_{u}$. This is true in particular for the sequence $\left(x_{n}, y_{n}\right)=\widetilde{T}_{u}^{n+1}(x, \infty)$. 
Also associated to $x$ is the sequence $\left(\theta_{n}, \theta_{n+1}\right)=F_{u}\left(x_{n}, y_{n}\right) \in \Omega_{u}$. Applying Fact 5 and Lemma 1 gives

$$
\begin{aligned}
\lim _{n \rightarrow \infty} \frac{1}{n} \#\left\{j \leq n \mid\left(\theta_{j}, \theta_{j+1}\right) \in D\right\} & =\lim _{n \rightarrow \infty} \frac{1}{n} \#\left\{j \leq n \mid\left(x_{j}, y_{j}\right) \in F_{u}^{-1}(D)\right\} \\
& =\rho_{u}\left(F_{u}^{-1}(D)\right)=\lambda_{u}(D) .
\end{aligned}
$$

To see the final statement of the theorem, define probability measures $P_{n}=\frac{1}{n} \sum_{j=1}^{n} \delta_{\left(\theta_{j}, \theta_{j+1}\right)}$ on $\Omega_{u}$, where $\delta_{(w, z)}$ is the point mass at $(w, z)$. It follows from the above that for almost all $x, \lim _{n \rightarrow \infty} P_{n}(D)=\lambda_{u}(D)$ for any Borel subset $D$ of $\Omega_{u}$ with boundary of measure zero. By the Portmanteau Theorem [4],

$$
\lim _{n \rightarrow \infty} \frac{1}{n} \sum_{j=1}^{n} f\left(\theta_{j}, \theta_{j+1}\right)=\lim _{n \rightarrow \infty} \int_{\Omega_{u}} f d P_{n}=\int_{\Omega_{u}} f d \lambda_{u}
$$

3.4. First application: a sum of Jager's. It follows immediately from Theorem 2 that for $u \in \Psi$ and almost all $x \in[0,1]$

$$
\lim _{n \rightarrow \infty} \frac{1}{n} \sum_{j=1}^{n}\left|\theta_{j}-\theta_{j+1}\right|=c_{u}|k| \int_{\Omega_{u}} \frac{|w-z|}{\sqrt{1-4 k z w}} d z d w
$$

which leads to:

Theorem 3. Suppose $|k|>1$. Then for almost all $x, \lim _{n \rightarrow \infty} \frac{1}{n} \sum_{j=1}^{n}\left|\theta_{j}-\theta_{j+1}\right|$
equals

$$
\begin{cases}\left(\log \frac{|k|+1}{|k|}\right)^{-1}\left(\frac{1}{|k|}-\frac{\sin ^{-1} \frac{2 \sqrt{|k|}}{|k|+1}}{2 \sqrt{|k|}}\right) & \text { if }(-1)^{m} k>0, \\ \left(\log \frac{|k|}{|k|-1}\right)^{-1}\left(-\frac{1}{|k|}+\frac{\sinh ^{-1} \frac{2 \sqrt{|k|}}{|k|-1}}{2 \sqrt{|k|}}\right) \quad \text { if }(-1)^{m} k<0 .\end{cases}
$$

Proof. The region $\Omega_{u}$ is symmetric about the line $z=w$ and the integrand in (8) is invariant under the symmetry. This means we can evaluate the integral by restricting attention to the lower half of $\Omega_{u}$ and then doubling the value. When $(-1)^{m} k>0$ and $|k| \geq 1$ the right-hand side of (8) becomes

$$
2|k| c_{u} \int_{0}^{\frac{1}{|k|+1}} \int_{z}^{\frac{-1}{|k|} z+\frac{1}{|k|}} \frac{w-z}{\sqrt{1-4|k| z w}} d w d z
$$


Using Mathematica very carefully we get

$$
\begin{aligned}
\left.2|k| c_{u} \int_{0}^{\frac{1}{|k|+1}}\left(\left(\frac{w}{6|k| z}-\frac{-1+6|k| z^{2}}{12|k|^{2} z^{2}}\right) \sqrt{1-4|k| z w}\right)\right|_{z} ^{\frac{-1}{|k|} z+\frac{1}{|k|}} d z \\
=2|k| c_{u} \int_{0}^{\frac{1}{|k|+1}} \frac{\left(1-4|k| z^{2}\right)^{3 / 2}+\sqrt{(1-2 z)^{2}}\left(6|k| z^{2}+2 z^{2}-2 z-1\right)}{12|k|^{2} z^{2}} d z \\
=2|k| c_{u} \frac{1}{12|k|^{2}}\left(-\left(\frac{1}{z}+2|k| z\right) \sqrt{1-4|k| z^{2}}\right. \\
+\sqrt{(1-2 z)^{2}}\left(6|k| z^{3}+2 z^{3}-6|k| z^{2}-6 z^{2}-1\right) \\
+c_{u}\left(\frac{\operatorname{sgn}(|k|-1)}{|k|}-\frac{1}{2 \sqrt{|k|}} \sin ^{-1} \frac{2 \sqrt{|k|}(2 \sqrt{|k|} z))}{1+|k|}\right)
\end{aligned}
$$

and the result follows.

When $(-1)^{m} k<0$ the right-hand side of (8) can be written as the sum of two integrals,

$$
\begin{aligned}
& 2|k| c_{u} \int_{0}^{\frac{1}{|k|}} \int_{0}^{w} \frac{w-z}{\sqrt{1-4|k| z w}} d z d w \\
& +2|k| c_{u} \int_{\frac{1}{|k|}}^{\frac{1}{|k|-1}} \int_{|k| w-1}^{w} \frac{w-z}{\sqrt{1-4|k| z w}} d z d w .
\end{aligned}
$$

The integrals are computed, as above, using Mathematica.

3.5. Second Application: the distribution of $\boldsymbol{\theta}_{\boldsymbol{n}}$. The next application of Theorem 2 is to derive the distribution of the sequence $\left\{\theta_{n}\right\}$ for generic $x$. It follows from Corollary 1 that for $u \in \Phi$ and for all $u$-irrational $x \in(0,1)$, $\theta_{n}$ lies in the interval

$$
\mathbf{I}_{u}= \begin{cases}\left(0, \frac{1}{|k|}\right) & \text { if }(-1)^{m} k>0, \\ \left(0, \frac{1}{|k|-1}\right) & \text { if }(-1)^{m} k<0 .\end{cases}
$$

Let $\phi: \Omega_{u} \rightarrow \mathbf{I}_{u}$ denote the projection $\phi(w, z)=w$. Define the measure $\beta_{u}$ on $\mathbf{I}_{u}$ by $\beta_{u}(D)=\lambda_{u}\left(\phi^{-1}(D)\right)$. Then it is clear that for almost all $x \in(0,1)$,

$$
\begin{aligned}
\beta_{u}(D) & =\lim _{n \rightarrow \infty} \frac{1}{n} \#\left\{j \leq n \mid\left(\theta_{n}, \theta_{n+1}\right) \in \phi^{-1}(D)\right\} \\
& =\lim _{n \rightarrow \infty} \frac{1}{n} \#\left\{j \leq n \mid \theta_{n} \in D\right\},
\end{aligned}
$$


and therefore $\beta_{u}$ describes the distribution of $\theta_{n}$ in $\mathbf{I}_{u} . \beta_{u}$ is equivalent to Lebesgue measure and the density $\beta_{u}(w)$ is obtained by projecting the density $\lambda_{u}(w, z)$ onto the $w$-axis by integration with respect to the $z$ variable. $\chi_{A}$ shall denote the characteristic function of the set $\mathrm{A}$. Then we have the following generalization of the Lenstra conjecture, originally proved by Bosma, Jager and Wiedijk [5]:

Theorem 4. For $u \in \Psi$ and for almost all $u$-irrational $x \in(0,1)$ the sequence $\theta_{n}, n=1,2, \ldots$, is distributed in the interval $\mathbf{I}_{u}$ according to the density function $\beta_{u}(w)$ defined by

$$
\left(\log \frac{|k|+1}{|k|}\right)^{-1}\left(\chi_{\left(0, \frac{1}{|k|+1}\right)}(w)+\left(\frac{1}{w}-|k|\right) \chi_{\left(\frac{1}{|k|+1}, \frac{1}{|k|}\right)}(w)\right)
$$

if $(-1)^{m} k>0$, and by

$$
\left(\log \frac{|k|}{|k|-1}\right)^{-1}\left(\chi_{\left(0, \frac{1}{|k|}\right)}(w)+\left(\frac{1}{w}+1-|k|\right) \chi_{\left(\frac{1}{|k|}, \frac{1}{|k|-1}\right)}(w)\right)
$$

if $(-1)^{m} k<0$.

Proof. We perform the calculations in the cases where $m=0$. When $m=1$ the computations run parallel to those presented below. First consider $0<$ $k<1$. The interval $\mathbf{I}_{u}=\left(0, \frac{1}{k}\right)$ is divided into four subintervals, over which the limits of integration will differ. For each of the two inner intervals it is necessary to consider separately the integration over pieces of the regions $\Omega_{u}^{*}$ and $\Lambda_{u}$ lying above the intervals. Refer to the first illustration of $\Omega_{u}$ in Figure 1 .

We thus have $\beta_{u}(w)=\mathrm{I}+\mathrm{II}+\mathrm{III}+\mathrm{IV}$, where

$$
\begin{aligned}
\mathrm{I} & =c_{u}\left(\int_{0}^{-\frac{1}{k} w+\frac{1}{k}} \frac{k}{\sqrt{1-4 k z w}} d z\right)\left(\chi_{\left(0, \frac{1}{2}\right)}(w)+\chi_{\left(\frac{1}{2}, \frac{1}{k+1}\right)}(w)\right) \\
& =c_{u}\left(\frac{-\sqrt{(2 w-1)^{2}}+1}{2 w}\right)\left(\chi_{\left(0, \frac{1}{2}\right)}(w)+\chi_{\left(\frac{1}{2}, \frac{1}{k+1}\right)}(w)\right), \\
\mathrm{II} & =c_{u}\left(\int_{-\frac{1}{k} w+\frac{1}{k}}^{\frac{1}{4 w k}} \frac{2 k}{\sqrt{1-4 k z w}} d z\right) \chi_{\left(\frac{1}{2}, \frac{1}{k+1}\right)}(w) \\
& =c_{u}\left(\frac{\sqrt{(2 w-1)^{2}}}{w}\right) \chi_{\left(\frac{1}{2}, \frac{1}{k+1}\right)}(w), \\
\mathrm{III} & =c_{u}\left(\int_{-k w+1}^{\frac{1}{4 w k}} \frac{2 k}{\sqrt{1-4 k z w}} d z\right) \chi_{\left(\frac{1}{k+1}, \frac{1}{2 k}\right)}(w) \\
& =c_{u}\left(\frac{\sqrt{(2 k w-1)^{2}}}{w}\right) \chi_{\left(\frac{1}{k+1}, \frac{1}{2 k}\right)}(w),
\end{aligned}
$$




$$
\begin{aligned}
\mathrm{IV} & =c_{u}\left(\int_{0}^{-k w+1} \frac{k}{\sqrt{1-4 k z w}} d z\right)\left(\chi_{\left(\frac{1}{k+1}, \frac{1}{2 k}\right)}(w)+\chi_{\left(\frac{1}{2 k}, \frac{1}{k}\right)}(w)\right) \\
& =c_{u}\left(\frac{-\sqrt{(2 k w-1)^{2}}+1}{2 w}\right)\left(\chi_{\left(\frac{1}{k+1}, \frac{1}{2 k}\right)}(w)+\chi_{\left(\frac{1}{2 k}, \frac{1}{k}\right)}(w)\right) .
\end{aligned}
$$

Thus

$$
\begin{aligned}
\beta_{u}(w)= & c_{u}\left(\frac{1-|2 w-1|}{2 w}\right) \chi_{\left(0, \frac{1}{2}\right)}(w)+c_{u}\left(\frac{1+|2 w-1|}{2 w}\right) \chi_{\left(\frac{1}{2}, \frac{1}{k+1}\right)}(w) \\
& +c_{u}\left(\frac{1+|2 k w-1|}{2 w}\right) \chi_{\left(\frac{1}{k+1}, \frac{1}{2 k}\right)}(w)+c_{u}\left(\frac{1-|2 k w-1|}{2 w}\right) \chi_{\left(\frac{1}{2 k}, \frac{1}{k}\right)}(w) \\
= & \left(\log \left|\frac{k+1}{k}\right|\right)^{-1} \chi_{\left(0, \frac{1}{k+1}\right)}(w)+\left(\log \left|\frac{k+1}{k}\right|\right)^{-1}\left(\frac{1}{w}-k\right) \chi_{\left(\frac{1}{k+1}, \frac{1}{k}\right)}(w),
\end{aligned}
$$

completing the argument in this case.

When $k>1, \beta_{u}(w)$ is the sum of the integrals I and IV above, or

$$
\beta_{u}(w)=c_{u}\left(\frac{1-|2 w-1|}{2 w}\right) \chi_{\left(0, \frac{1}{k+1}\right)}(w)+c_{u}\left(\frac{1-|2 k w-1|}{2 w}\right) \chi_{\left(\frac{1}{k+1}, \frac{1}{k}\right)}(w) .
$$

Then $|2 w-1|=1-2 w$ for $0<w<\frac{1}{k+1}$ and $|2 k w-1|=2 k w-1$ for $\frac{1}{k+1}<w<\frac{1}{k}$. The result follows.

When $m=0$ and $k<-1$, the region $\Omega$, pictured to the right in Figure 1, divides naturally into two pieces: one above the interval $\left(0, \frac{1}{|k|}\right)$ and another to the right above the interval $\left(\frac{1}{|k|}, \frac{1}{|k|-1}\right)$. Then

$$
\begin{aligned}
\beta_{u}(w)=c_{u} \chi_{\left(0, \frac{1}{|k|}\right)}(w) \int_{0}^{-\frac{1}{k} w-\frac{1}{k}} & \frac{|k|}{\sqrt{1-4 k z w}} d z \\
& +c_{u} \chi_{\left(\frac{1}{|k|}, \frac{1}{|k|-1}\right)}(w) \int_{-k w-1}^{-\frac{1}{k} w-\frac{1}{k}} \frac{|k|}{\sqrt{1-4 k z w}} d z .
\end{aligned}
$$

Computing, as above, gives

$$
\beta_{u}(w)=c_{u} \chi_{\left(0, \frac{-1}{k}\right)}(w)+c_{u}\left(\frac{1}{w}+1+k\right) \chi_{\left(-\frac{1}{k},-\frac{1}{k+1}\right)}(w) .
$$

Here is an immediate application of Theorem 4:

Theorem 5. For $u \in \Psi$ and almost all $x \in(0,1)$,

$$
\lim _{n \rightarrow \infty} \frac{1}{n} \sum_{i=1}^{n} \theta_{i}(x)= \begin{cases}\left(2\left(k^{2}+|k|\right) \log \frac{|k|+1}{|k|}\right)^{-1} & \text { if }(-1)^{m} k>0, \\ \left(2\left(k^{2}-|k|\right) \log \frac{|k|}{|k|-1}\right)^{-1} & \text { if }(-1)^{m} k<0 .\end{cases}
$$


Proof. For almost all $x$

$$
\lim _{n \rightarrow \infty} \frac{1}{n} \sum_{i=1}^{n} \theta_{i}(x)=\int_{\mathbf{I}_{u}} w \beta_{u}(w) d w .
$$

Computing the integral in the various cases proves the theorem.

\section{References}

[1] L. Ahlfors, Complex Analysis: An Introduction to the Theory of Analytic Functions of one Complex Variable, International Series in Pure and Applied Mathematics, McGraw-Hill, New York, 1979, MR 0510197 (80c:30001), Zbl 0395.30001.

[2] T. Bedford, M. Keane, C. Series, eds., Ergodic Theory, Symbolic Dynamics and Hyperbolic Spaces, Papers from the Workshop on Hyperbolic Geometry and Ergodic Theory held in Trieste, April 17-28, 1989, Oxford Science Publications, Oxford Univ. Press, New York, 1991, MR 1130170 (93e:58002), Zbl 0743.00040.

[3] P. Billingsley, Ergodic Theory and Information, Wiley, New York-London, 1965, MR 0192027 (33 \#254), Zbl 0141.16702.

[4] P. Billingsley, Convergence of Probability Measures, Wiley, New York-London, 1968, MR 0233396 (38 \#1718), Zbl 0172.21201.

[5] W. Bosma, H. Jager and F. Wiedijk, Some metrical observations on the approximation of continued factions, Indag. Math., 45 (1983), 281-299, MR 0718069 (85f:11059), Zbl 0519.10043.

[6] I.P. Cornfeld, S.V. Fomin and Ya.G. Sinai, Ergodic Theory, Grundlehren der Mathematischen Wissenschaften, 245, Springer-Verlag, New York, 1982, MR 0832433 (87f:28019), Zbl 0493.28007.

[7] K. Gröchenig and A. Haas, Backward continued fractions, Hecke groups and invariant measures for transformations of the interval, Ergodic Theory Dynam. Systems, 16 (1996), 1241-1274, MR 1424398 (97m:58114), Zbl 0884.58040.

[8] A. Haas, Invariant measures and natural extensions, Canad. Math. Bull., 45 (2002), 97-108, MR 1884139 (2002j:37005).

[9] A. Haas and D. Molnar, Metrical diophantine approximation for continued fraction like mappings of the interval, Trans. Amer. Math. Soc., 356 (2004), 2851-2870, MR 2052599.

[10] H. Jager, The distribution of certain sequences connected with the continued fraction, Indag. Math., 89 (1986), 61-69, MR 0834320 (87g:11092), Zbl 0588.10061.

Received October 4, 2002.

Department of Mathematics

The University of Connecticut

STORRS CT 06269-3009

E-mail address: haas@math.uconn.edu

Department of Mathematics \& Computer Science

Gustavus Adolphus College

St. Peter MN 56082

E-mail address: dmolnar@gac.edu 